\title{
Probing the trilinear Higgs boson coupling in di-Higgs production at NLO QCD including parton shower effects
}

\author{
G. Heinrich, ${ }^{a}$ S. P. Jones, ${ }^{b}$ M. Kerner, ${ }^{c}$ G. Luisoni ${ }^{a}$ and L. Scyboz ${ }^{a}$ \\ ${ }^{a}$ Max Planck Institute for Physics, \\ Föhringer Ring 6, 80805 München, Germany \\ ${ }^{b}$ Theoretical Physics Department, CERN, \\ Geneva, Switzerland \\ ${ }^{c}$ Physik-Institut, Universität Zürich, \\ Winterthurerstrasse 190, 8057 Zürich, Switzerland \\ E-mail: gudrun@mpp.mpg.de, s.jones@cern.ch, mkerner@physik.uzh.ch, \\ luisonig@gmail.com, scyboz@mpp.mpg.de
}

ABSTRACT: We present results for Higgs boson pair production with variations of the trilinear Higgs boson self coupling at next-to-leading order (NLO) in QCD including the full top quark mass dependence. Differential results for the LHC at $14 \mathrm{TeV}$ are presented and we discuss the implications of anomalous trilinear couplings as well as differences between the Pythia and Herwig parton showers in combination with POWHEG. The implementation of the NLO QCD calculation with variable Higgs boson self coupling is made publicly available in the POWHEG-BOX-V2 Monte Carlo framework. A simple method for using the new implementation to study also variations of the top quark Yukawa coupling is described.

Keywords: NLO Computations, QCD Phenomenology

ARXIV EPRINT: 1903.08137 


\section{Contents}

1 Introduction 1

2 Overview of the calculation 3

3 Total and differential cross sections at non-SM trilinear couplings $\quad 5$

3.1 Total cross sections at different values of the trilinear coupling 6

$\begin{array}{lll}3.2 & \text { Differential cross sections } & 6\end{array}$

$\begin{array}{lll}3.3 & \text { Discussion of parton shower related uncertainties } & 7\end{array}$

4 Conclusions 10

\section{Introduction}

The Higgs potential is currently the least explored part of the Standard Model (SM), measurements of the Higgs boson self-coupling(s) may therefore offer surprises. Although the Higgs boson couplings to vector bosons and third generation fermions are increasingly well measured [1-5], constraints on the trilinear coupling $\lambda$ are relatively weak due to the small Higgs boson pair production cross sections $[6,7]$. Nonetheless, measurements of double Higgs production in gluon fusion, combining various decay channels, have led to impressive experimental results already [8-11], the most stringent constraints on the trilinear coupling being $-5 \leq \kappa_{\lambda} \leq 12.1$ at $95 \%$ confidence level [10], based on the assumption that all other couplings have SM values. Individual limits on $\kappa_{\lambda}$ based on EFT benchmarks representing a certain combination of BSM couplings which leads to characteristic kinematic distributions [12-14] have also been extracted [8, 9]. Therefore, the determination of the trilinear coupling has entered a level of precision where the assumption that the full NLO QCD corrections do not vary much with $\kappa_{\lambda}$, which has been used in the experimental analysis so far, needs to be revised. The variations of the K-factors with $\kappa_{\lambda}$ are mild in the $m_{t} \rightarrow \infty$ limit, where NLO [15, 16] and NNLO [17] corrections have been calculated within an effective Lagrangian framework. However, it will be shown in this paper that the NLO K-factor varies by about $35 \%$ as $\kappa_{\lambda}$ is varied between -1 and 5 once the full top quark mass dependence is taken into account.

The question of how large or small $\kappa_{\lambda}$ can be from a theory point of view is not easy to answer in a model independent way. Recent work based on rather general concepts like vacuum stability and perturbative unitarity suggests that $\left|\kappa_{\lambda}\right| \lesssim 4$ for a New Physics scale in the few TeV range [18-21]. More specific models can lead to more stringent bounds, see e.g. refs. [22-25]. Recent phenomenological studies about the precision that could be reached for the trilinear coupling at the (HL-)LHC and future hadron colliders can be found for example in refs. [26-36]. 
Higgs boson pair production in gluon fusion in the SM has been calculated at leading order in refs. [37-39]. The NLO QCD corrections with full top quark mass dependence became available more recently [40-42]. The NLO results of refs. [40, 41] have been supplemented by soft-gluon resummation at small transverse momenta of the Higgs boson pair [43] and by parton shower effects [44, 45]. Before the full NLO QCD corrections became available, the $m_{t} \rightarrow \infty$ limit, sometimes also called Higgs Effective Field Theory (HEFT) approximation, has been used in several forms of approximations. In this limit, the NLO corrections were first calculated in ref. [46] using the so-called "Born-improved HEFT" approximation, which involves rescaling the NLO results in the $m_{t} \rightarrow \infty$ limit by a factor $B_{\mathrm{FT}} / B_{\mathrm{HEFT}}$, where $B_{\mathrm{FT}}$ denotes the LO matrix element squared in the full theory. In ref. [47] an approximation called "FT approx", was introduced, which contains the real radiation matrix elements with full top quark mass dependence, while the virtual part is calculated in the Born-improved HEFT approximation.

The NNLO QCD corrections in the $m_{t} \rightarrow \infty$ limit have been computed in refs. [4851]. These results have been improved in various ways: they have been supplemented by an expansion in $1 / m_{t}^{2}$ in [52], and soft gluon resummation has been performed at NNLO+NNLL level in [53]. The calculation of ref. [51] has been combined with results including the top quark mass dependence as far as available in ref. [54], and the latter has been supplemented by soft gluon resummation in ref. [55].

The scale uncertainties at NLO are still at the $10 \%$ level, while they are decreased to about $5 \%$ when including the NNLO corrections. The uncertainties due to the chosen top mass scheme have been assessed in ref. [42], where the full NLO corrections, including the possibility to switch between pole mass and $\overline{\mathrm{MS}}$ mass, have been presented.

Analytic approximations for the top quark mass dependence of the two-loop amplitudes in the NLO calculation have been studied in refs. [56-59] and complete analytic results in the high energy limit have been presented in ref. [60]. The formalism of an expansion for large top quark mass has been applied recently to calculate partial real-radiation corrections to Higgs boson pair production at NNLO in QCD [61].

In this work we study the dependence of total cross sections and differential distributions on the trilinear Higgs boson coupling, assuming that the BSM-induced deviations in the other couplings are at the (sub-)percent level. The study is based on results at NLO QCD with full top quark mass dependence for Higgs boson pair production in gluon fusion described in refs. [40, 41]. While it is unlikely that New Physics alters just the Higgs boson self-couplings but leaves the Higgs couplings to vector bosons and fermions unchanged, it can be assumed that the deviations of the measured Higgs couplings from their SM values are so small that they have escaped detection at the current level of precision, for recent overviews see e.g. refs. [26, 62-64].

Measuring Higgs boson pair production is a direct way to access the trilinear Higgs coupling. The trilinear and quartic couplings can also be constrained in an indirect way, through measurements of processes which are sensitive to the Higgs boson self-couplings via electroweak corrections [28, 65-77]. Such processes offer important complementary information, however they are more susceptible to other BSM couplings entering the loop corrections at the same level, and therefore the limits on $\kappa_{\lambda}$ extracted this way may be more 
model dependent than the ones extracted from the direct production of Higgs boson pairs.

For Higgs Boson pair production, due to the destructive interference in the squared amplitude between contributions containing $\lambda$ and those without the Higgs boson selfcoupling (corresponding to triangle- and box diagrams, respectively, at LO), small changes in $\lambda$ modify the interference pattern and can therefore have a substantial effect on the total cross section and differential distributions.

In order to obtain a fully-fledged NLO generator which also offers the possibility of parton showering, we have implemented our calculation in the POWHEG-BOX [78-80], building on the SM code presented in ref. [44].

The dependence of the K-factors on the value of $\lambda$ (and other BSM couplings) is stronger than the $m_{t} \rightarrow \infty$ limit may suggest, as shown in ref. [14]. This is particularly true for differential K-factors. For example, in the boosted regime, which is sometimes used by the experiments when reconstructing the $H \rightarrow b \bar{b}$ decay channel, Higgs bosons with a large- $p_{T}$ are involved. At large- $p_{T}$ the top quark loops are resolved and the $m_{t} \rightarrow \infty$ limit is invalid. The top quark mass corrections in the large $m_{\mathrm{hh}}$ or $p_{T}^{\mathrm{h}}$ regime are of the order of $20-30 \%$ or higher, and increase with larger centre-of-mass energy (e.g. $\sqrt{s}=27$ (HE-LHC) or $100 \mathrm{TeV}$ (FCC-hh)), these corrections clearly exceed the scale uncertainties and therefore have to be taken into account.

The purpose of this paper is twofold: based on our differential results, we discuss how the deviations from the SM, resulting from non-SM $\lambda$ values, can be identified based on the distributions for the Higgs boson pair invariant mass and Higgs boson transverse momentum distributions. In addition, we present the updated public code POWHEG-BOX-V2/ggHH, where the user can choose the value of the trilinear coupling as an input parameter. We also explain how variations of the top-Higgs Yukawa coupling can be studied using this code. Further, we compare the fixed order results to results obtained by matching the NLO calculation to a parton shower. In particular, we compare results from the Pythia 8.2 [81] and Herwig 7.1 [82] parton showers and assess the parton-shower related uncertainties.

This paper is organised as follows. In section 2 we briefly describe the calculation and give instructions for the usage of the program within the POWHEG-BOX. Section 3 contains the discussion of our results, focusing in the first part on variations of $\kappa_{\lambda}$ and in the second part on differences between showered results. We present our conclusions in section 4 .

\section{Overview of the calculation}

The calculation builds on the one presented in ref. [44] and therefore will be described only briefly here.

The leading order amplitude in the full theory and all the amplitudes in the $m_{t} \rightarrow \infty$ limit were implemented analytically, whereas the one-loop real radiation contribution and the two-loop virtual amplitudes in the full SM rely on numerical or semi-numerical codes. The real radiation matrix elements in the full SM were implemented using the interface [83] between GoSAM $[84,85]$ and the POWHEG-BOX [78-80], modified accordingly to compute the real corrections to the loop-induced Born amplitude. At run time the amplitudes were computed using Ninja [86], golem95C [87, 88] and OneLOop [89] for the evaluation of the 
scalar one-loop integrals. The stability of the amplitudes in the collinear limits has been improved by a better detection of instabilities in the real radiation and the use of the scalar four-point function from VBFNLO [90, 91].

For the virtual corrections, containing two-loop amplitudes, we have used the results of the calculation presented in refs. [40, 41], which used also Reduze 2 [92] and SEcDEc 3 [93].

The values for the Higgs boson and top quark masses have been set to $m_{h}=125 \mathrm{GeV}$ and $m_{t}=173 \mathrm{GeV}$, such that the two-loop amplitudes are only functions of two independent variables, the parton-level Mandelstam invariants $\hat{s}$ and $\hat{t}$. We have constructed a grid in these variables, based on 5291 pre-computed phase-space points, together with an interpolation framework, such that an external program can call the virtual two-loop amplitude at any phase space point without having to do costly two-loop integrations. We used the same setup for the grid as described in ref. [44] and extended it in the following way: we can write the squared matrix element as a polynomial of degree two in $\lambda$,

$$
M_{\lambda} \equiv\left|\mathcal{M}_{\lambda}\right|^{2}=A+B \lambda+C \lambda^{2} .
$$

Therefore it is sufficient to know the amplitude at three different values of $\lambda$ in order to reconstruct the full $\lambda$-dependence. Choosing $\lambda=-1,0,1$ we obtain

$$
A=M_{0}, B=\left(M_{1}-M_{-1}\right) / 2, C=\left(M_{1}+M_{-1}\right) / 2-M_{0} .
$$

In practice we used the representation

$$
M_{\lambda}=M_{0}\left(1-\lambda^{2}\right)+\frac{M_{1}}{2}\left(\lambda+\lambda^{2}\right)+\frac{M_{-1}}{2}\left(-\lambda+\lambda^{2}\right)
$$

in order to get a more straightforward uncertainty estimate.

In fact, to any order in QCD, we can separate the matrix element into a piece that depends only on the top quark Yukawa coupling $y_{t}$ ("box diagrams") and a piece that depends on the Higgs boson trilinear self-coupling $\lambda$ ("triangle diagrams"):

$$
\mathcal{M}=y_{t}^{2} \mathcal{M}_{B}+y_{t} \lambda \mathcal{M}_{T}
$$

The squared amplitude at each order can then be written as

$$
|\mathcal{M}|^{2}=y_{t}^{4}\left[\mathcal{M}_{B} \mathcal{M}_{B}^{*}+\frac{\lambda}{y_{t}}\left(\mathcal{M}_{B} \mathcal{M}_{T}^{*}+\mathcal{M}_{T} \mathcal{M}_{B}^{*}\right)+\frac{\lambda^{2}}{y_{t}^{2}} \mathcal{M}_{T} \mathcal{M}_{T}^{*}\right]
$$

The above parametrisation makes it clear that the dependence of the cross section on both the Yukawa coupling and the Higgs boson self-coupling can be reconstructed from only the 3 terms present in eq. (2.1). Of course this pattern changes once electroweak corrections, part of which have been calculated recently [28,67], are included.

In order to allow for comparisons and cross checks, we implemented both the $m_{t} \rightarrow \infty$ limit as well as the amplitudes with full $m_{t}$-dependence at NLO. This allows to run the code in four different modes by changing the flag mtdep in the POWHEG-BOX run card. The possible choices are the following: 
mtdep $=0$ : computation using basic HEFT: all amplitudes are computed in the $m_{t} \rightarrow \infty$ limit.

mtdep=1: computation using Born-improved HEFT. In this approximation the NLO part is computed in the $m_{t} \rightarrow \infty$ limit and reweighted pointwise in the phase-space by the ratio of the LO matrix element with full mass dependence to the LO matrix element in HEFT.

mtdep=2: computation in the approximation $\mathrm{FT}_{\text {approx }}$. In this approximation the matrix elements for the Born and the real radiation contributions are computed with full top quark mass dependence, whereas the virtual part is computed as in the Bornimproved HEFT case.

mtdep=3: computation with full top quark mass dependence.

Detailed instructions on how to run the code can be found in the file manual-BOX-HH.pdf in the folder ggHH/Docs of the program.

When mtdep $=3$ is selected, the result of the virtual matrix element is based on a grid of pre-sampled phase-space points as described above. The phase-space points present in the grid are distributed such that they optimally sample the Standard Model (SM) Born matrix element. The same set of points is used regardless of the value of $\lambda$ selected. Due to the finite number of points present in the grid, there is an associated statistical uncertainty which amounts to $0.1 \%$ on the total cross section at $14 \mathrm{TeV}$ for $\lambda=\lambda_{\mathrm{SM}}$. However, for $\lambda \neq \lambda_{\mathrm{SM}}$ the virtual matrix element can differ significantly in shape from the SM prediction, as is apparent from examining the $m_{\mathrm{hh}}$ and $p_{T}^{\mathrm{h}}$ distributions for different values of the Higgs boson self coupling. The uncertainty associated with the use of the grid is therefore larger for non-SM values of $\lambda$. The uncertainty increases as $\lambda$ is decreased below the SM value reaching $0.6 \%$ on the total cross section at $14 \mathrm{TeV}$ for $\kappa_{\lambda}=-1$. Increasing $\lambda$ above the SM value, we obtain an uncertainty of $3 \%$ on the total cross section at $14 \mathrm{TeV}$ for $\kappa_{\lambda}=3$ and $\kappa_{\lambda}=5$. Furthermore, for differential distributions the total uncertainty is not distributed uniformly in each bin but instead increases when the shape of the matrix element most differs from the SM prediction. Focusing on the invariant mass distribution, amongst the values of the Higgs boson self-coupling considered here, the largest uncertainty is obtained for the smallest values of $m_{\mathrm{hh}}$ and $\kappa_{\lambda}=3$. The uncertainty reaches $6 \%$ for the lowest bin when a $40 \mathrm{GeV}$ bin width is used.

\section{Total and differential cross sections at non-SM trilinear couplings}

The results were obtained using the PDF4LHC15_nlo_30_pdfas [94-97] parton distribution functions interfaced to our code via LHAPDF [98], along with the corresponding value for $\alpha_{s}$. The masses of the Higgs boson and the top quark have been fixed, as in the virtual amplitude, to $m_{h}=125 \mathrm{GeV}, m_{t}=173 \mathrm{GeV}$ and their widths have been set to zero. The top quark mass in renormalised in the on-shell scheme. Jets are clustered with the anti- $k_{T}$ algorithm [99] as implemented in the fastjet package [100, 101], with jet radius $R=0.4$ and a minimum transverse momentum $p_{T, \min }^{\text {jet }}=20 \mathrm{GeV}$. The scale 


\begin{tabular}{|c|c|c|c|c|}
\hline$\lambda_{\mathrm{BSM}} / \lambda_{\mathrm{SM}}$ & $\sigma_{\mathrm{NLO}} @ 13 \mathrm{TeV}[\mathrm{fb}]$ & $\sigma_{\mathrm{NLO}} @ 14 \mathrm{TeV}[\mathrm{fb}]$ & $\sigma_{\mathrm{NLO}} @ 27 \mathrm{TeV}[\mathrm{fb}]$ & K-factor@14TeV \\
\hline-1 & $116.71_{-14.3 \%}^{+16.4 \%}$ & $136.91_{-13.9 \%}^{+16.4 \%}$ & $504.9_{-11.8 \%}^{+14.1 \%}$ & 1.86 \\
\hline 0 & $62.51_{-13.7 \%}^{+15.8 \%}$ & $73.64_{-13.4 \%}^{+15.4 \%}$ & $275.29_{-11.3 \%}^{+13.2 \%}$ & 1.79 \\
\hline 1 & $27.84_{-12.9 \%}^{+11.6 \%}$ & $32.88_{-12.5 \%}^{+13.5 \%}$ & $127.7_{-10.4 \%}^{+11.5 \%}$ & 1.66 \\
\hline 2 & $12.42_{-12.0 \%}^{+13.1 \%}$ & $14.75_{-11.8 \%}^{+12.0 \%}$ & $59.10_{-9.7 \%}^{+10.2 \%}$ & 1.56 \\
\hline 2.4 & $11.65_{-12.7 \%}^{+13.9 \%}$ & $13.79_{-12.5 \%}^{+13.5 \%}$ & $53.67_{-10.3 \%}^{+11.4 \%}$ & 1.65 \\
\hline 3 & $16.28_{-15.3 \%}^{+16.2 \%}$ & $19.07_{-14.1 \%}^{+17.1 \%}$ & $69.84_{-12.1 \%}^{+14.6 \%}$ & 1.90 \\
\hline 5 & $81.74_{-15.6 \%}^{+20.0 \%}$ & $95.22_{-1.5 \%}^{+19.7 \%}$ & $330.61_{-13.6 \%}^{+17.4 \%}$ & 2.14 \\
\hline
\end{tabular}

Table 1. Total cross sections for Higgs boson pair production at full NLO QCD. The given uncertainties are scale uncertainties.

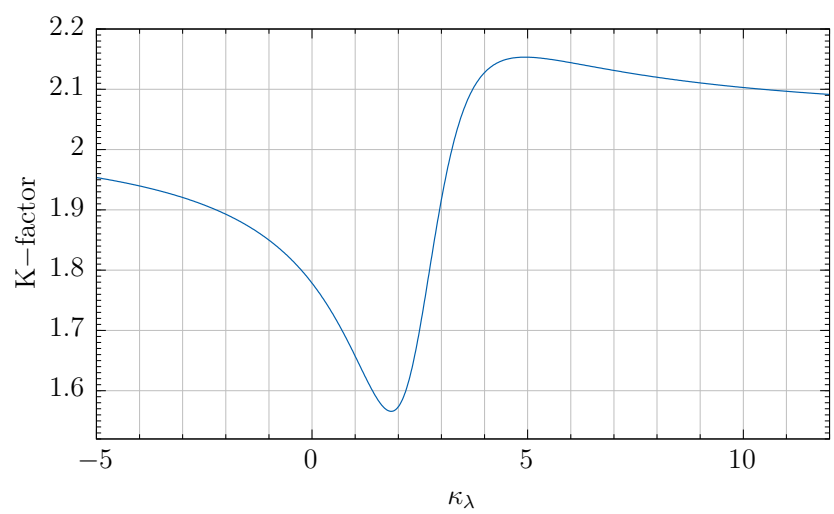

Figure 1. Variation of the NLO K-factor with the trilinear coupling at $\sqrt{s}=14 \mathrm{TeV}$.

uncertainties are estimated by varying the factorisation/renormalisation scales $\mu_{F}, \mu_{R}$. The scale variation bands represent scale variations around the central scale $\mu_{0}=m_{\mathrm{hh}} / 2$, with $\mu_{R}=\mu_{F}=c \mu_{0}$, where $c \in\{0.5,1,2\}$. For the case $\lambda=\lambda_{\mathrm{SM}}$ we checked that the bands obtained from these variations coincide with the bands resulting from 7-point scale variations. The PDF uncertainties have been studied in [64] and found to be in general considerably smaller than the scale uncertainties.

\subsection{Total cross sections at different values of the trilinear coupling}

In table 1 we list total cross sections at 13,14 and $27 \mathrm{TeV}$ for various values of the trilinear Higgs coupling $\lambda$. Table 1 also shows that the K-factors vary substantially as functions of the trilinear coupling. This fact is illustrated in figure 1, showing that the K-factor takes values between 1.56 and 2.15 if the trilinear coupling is varied between $-5 \leq \kappa_{\lambda} \leq 12$.

\subsection{Differential cross sections}

In figure 2 we show the $m_{\mathrm{hh}}$ distribution for various values of $\kappa_{\lambda}=\lambda_{\mathrm{BSM}} / \lambda_{\mathrm{SM}}$. The ratio plots show the ratio to the result with $\lambda_{\mathrm{SM}}$. A characteristic dip develops in the $m_{\mathrm{hh}}$ distribution around $\kappa_{\lambda}=2.4$, which is the value of maximal destructive interference between diagrams containing the trilinear coupling (triangle-type contributions) and "background" 


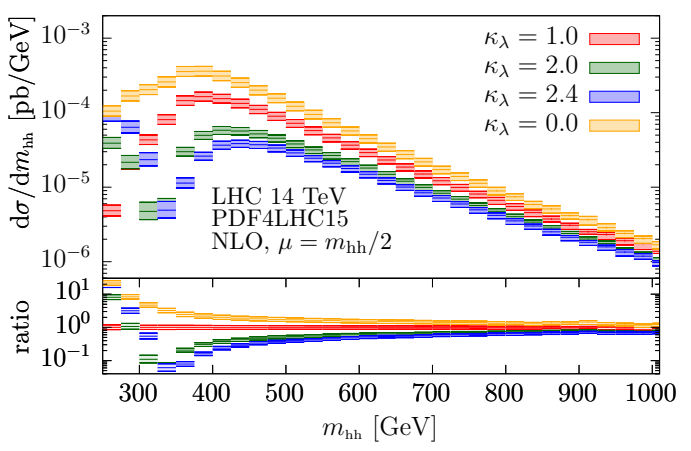

(a)

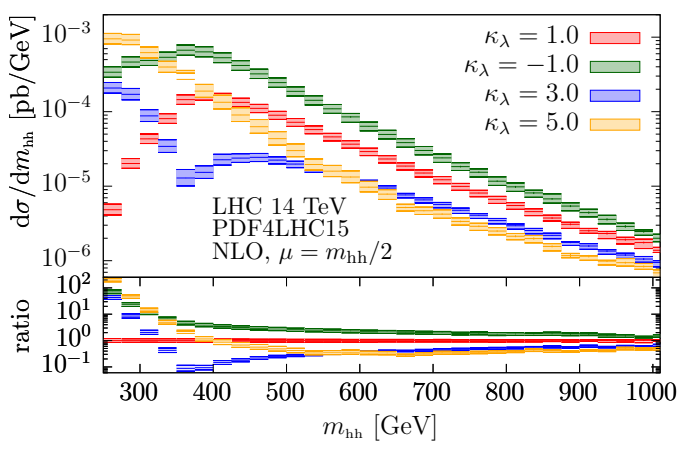

(b)

Figure 2. Higgs boson pair invariant mass distributions for various values of $\kappa_{\lambda}$ at $\sqrt{s}=14 \mathrm{TeV}$. The uncertainty bands are from scale variations as described in the text.

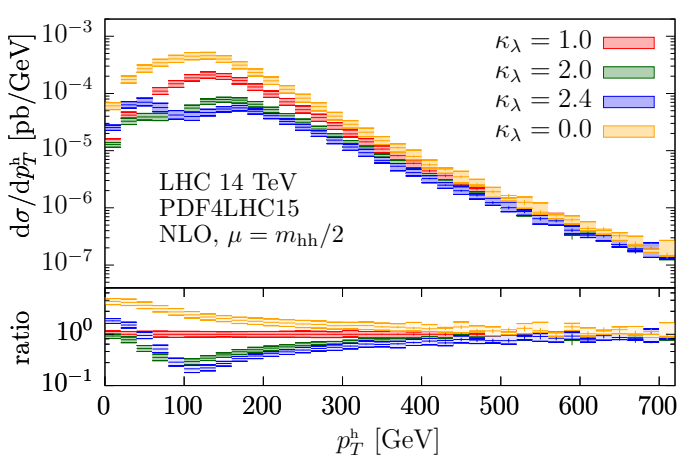

(a)

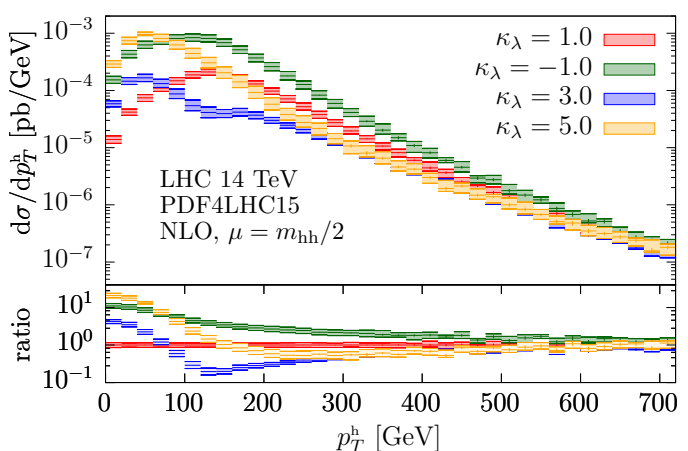

(b)

Figure 3. Higgs boson transverse momentum distributions for various values of $\kappa_{\lambda}$ at $\sqrt{s}=14 \mathrm{TeV}$.

diagrams (box-type contributions). Therefore we provide results for a denser spacing of $\kappa_{\lambda}$ values around this point.

In figure 3 we show the transverse momentum distributions $p_{T}^{h}$ of one (any) Higgs boson for different $\kappa_{\lambda}$ values. The dip for $\kappa_{\lambda} \sim 2.4$ is still present, however much less pronounced than in the $m_{\mathrm{hh}}$ distribution.

Figure 4 demonstrates the effect of variations of the top quark Yukawa coupling $y_{t}$ on the $m_{\mathrm{hh}}$ and $p_{T}^{\mathrm{h}}$ distributions, where $\kappa_{\lambda}$ is fixed to the SM value. Using eq. (2.5), it is apparent that $y_{t}$ variations can be obtained from appropriate $\kappa_{\lambda}$ variations with the same code. For example, $\sigma\left(y_{t}=1.2, \kappa_{\lambda}=1\right)=(1.2)^{4} \sigma\left(y_{t}=1, \kappa_{\lambda}=1 / 1.2\right)$.

\subsection{Discussion of parton shower related uncertainties}

In this section we show distributions for NLO results matched to a parton shower, focusing mostly on the transverse momentum of the Higgs boson pair. For this distribution NLO is the first non-trivial order, and therefore it is particularly sensitive to differences in the treatment of radiation by the parton shower. We compare the Pythia 8.2 [81] and Herwig 7.1 [82] parton showers, applied directly to the POWHEG Les Houches events (LHE). 


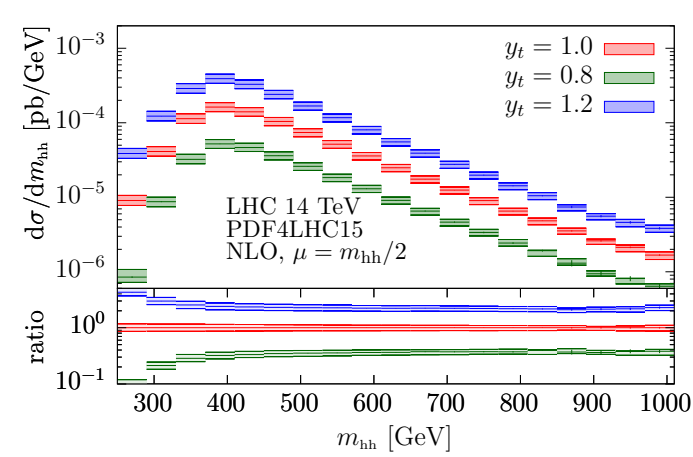

(a)

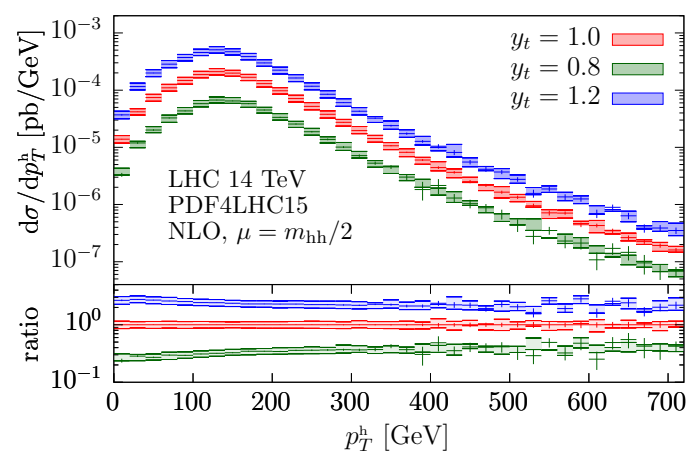

(b)

Figure 4. Higgs boson pair invariant mass distributions, and distributions of the transverse momentum of one (any) Higgs boson for non-SM values of the top quark Yukawa coupling $y_{t}$ at $\sqrt{s}=14 \mathrm{TeV}$, including scale uncertainties.

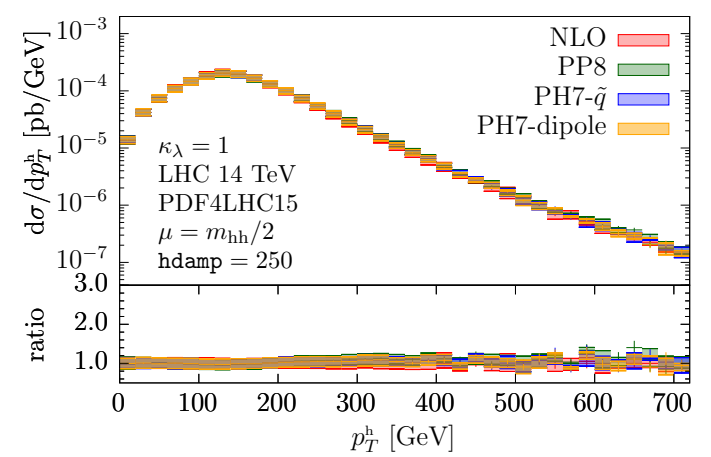

(a)

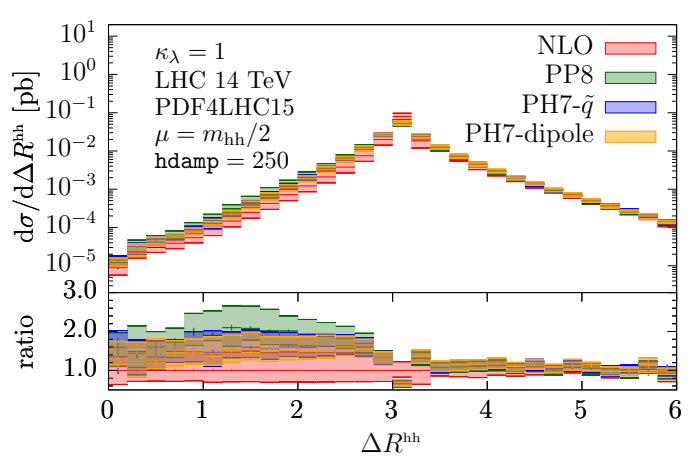

(b)

Figure 5. The transverse momentum of one (any) Higgs boson and the $R$-separation between the two Higgs bosons are shown for the fixed-order NLO calculation and three shower setups, in the $\kappa_{\lambda}=1$ case.

In the Herwig case, we also compare the default shower (the angular-ordered $\tilde{q}$-shower) with the dipole shower. In addition, we assess the uncertainties stemming from the matching and show results where the Herwig shower scale parameter HardScale is varied. For all shower algorithms considered, the default tune of the corresponding version is used. Multipleparton interactions (MPI) and hadronisation are switched off. The hdamp parameter in POWHEG is set to hdamp $=250 \mathrm{GeV}$.

In general, observables that are inclusive in the additional radiation, like the transverse momentum of one (any) Higgs boson, $p_{T}^{h}$, show little sensitivity to the details of the parton showering, as can be seen from figure 5(a), showing the fixed-order NLO prediction, as well as the Pythia 8.2 (PP8) and both Herwig 7.1 showers (angular-ordered PH7- $\tilde{q}$, and PH7-dipole). In contrast, figure 5(b) displays the distribution of the distance $\Delta R^{\mathrm{hh}}=$ $\sqrt{\left(\eta_{1}-\eta_{2}\right)^{2}+\left(\Phi_{1}-\Phi_{2}\right)^{2}}$ between the two Higgs bosons. There, the Sudakov exponent and the parton shower effectively resum the fixed-order prediction in the region where the two Higgs bosons are close to a back-to-back configuration, and the parton shower increases the fixed-order real radiation contribution in the region $\Delta R^{\mathrm{hh}}<\pi$. 


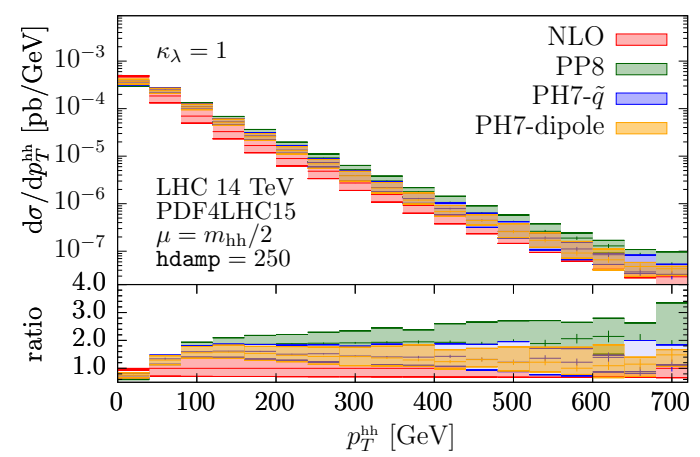

(a)

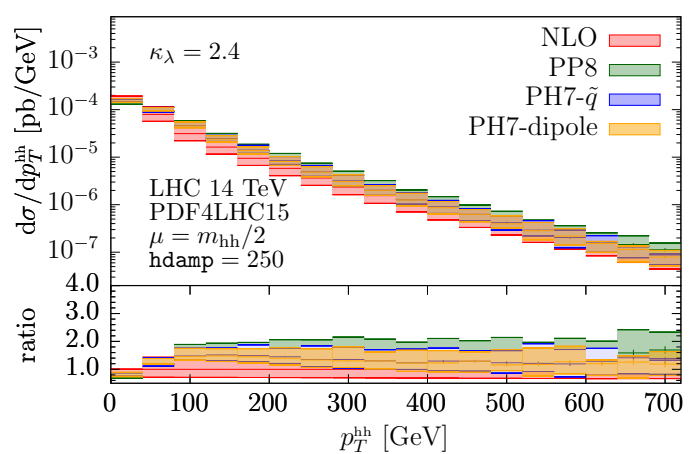

(b)

Figure 6. Transverse momentum of the Higgs boson pair for the fixed-order NLO calculation and all three shower setups at $14 \mathrm{TeV}$ for (a) $\kappa_{\lambda}=1$, (b) $\kappa_{\lambda}=2.4$.

In figures $6(\mathrm{a})$ and $6(\mathrm{~b})$, the transverse momentum $p_{T}^{\text {hh }}$ of the Higgs boson pair system is shown for the fixed-order and parton-showered predictions, at $\kappa_{\lambda}=1$ and $\kappa_{\lambda}=2.4$. In all cases, the Pythia and Herwig showers agree very well in the small- $p_{T}^{\mathrm{hh}}$ range, but start to deviate already at $p_{T}^{\text {hh }} \sim 100 \mathrm{GeV}$. While both Herwig showers give very similar results and reproduce the fixed-order calculation at high- $p_{T}^{\mathrm{hh}}$, the Pythia shower produces much harder additional radiation and the ratio to the fixed-order result plateaus at $\sim 2.0$ over the remaining range. We should mention that rather large differences between Pythia 8.2 and Herwig 7.1 showers matched to POWHEG also have been found studying top quark pair production [102]. The origin of the large NLO parton shower matching uncertainties affecting certain observables in Higgs boson pair production have previously been studied in literature [45]. For the SM result, the excess at large $p_{T}^{\text {hh }}$ produced when using POWHEG with Pythia 8.2 was found to be due to additional hard sub-leading jets generated purely by the shower [103].

With the Herwig default shower, systematic uncertainties can be estimated by varying the maximal transverse momentum allowed for shower emissions, by changing the so-called hard scale $\mu_{Q}$. We apply a factor $c_{Q}=\{0.5,2.0\}$ on the central hard shower scale, separately for all variations of the factorisation/renormalisation scales $\mu_{R, F}$. Figure 7 shows the $p_{T}^{\mathrm{hh}}$ and $\Delta R^{\mathrm{hh}}$ distributions as examples of the SM case, $\kappa_{\lambda}=1$, and underlines their sensitivity to changes in the shower hard scale. Quantitatively, the hard scale variations inflate the sole factorisation/renormalisation scale uncertainties by a factor of two in the regions where the Herwig 7.1 and Pythia 8.2 showers were in disagreement (see figures 5(b) and 6). If the envelope of all scale variations, including the hard shower scale, was to be taken as a theoretical systematic uncertainty, the resulting uncertainty would be of the order of $50 \%$ in these bins. It would be enlightening to further study parton shower (and non-perturbative) effects, in the particular context of Higgs boson pair production at NLO, as well as for loop-induced colour singlet production in general, and try to reduce discrepancies among the different algorithms. 


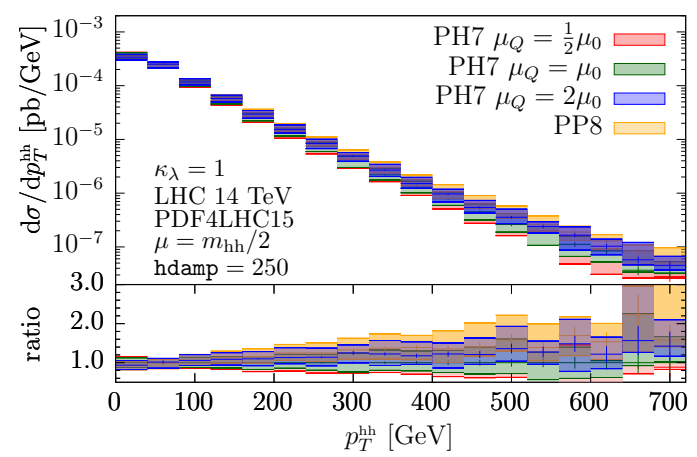

(a)

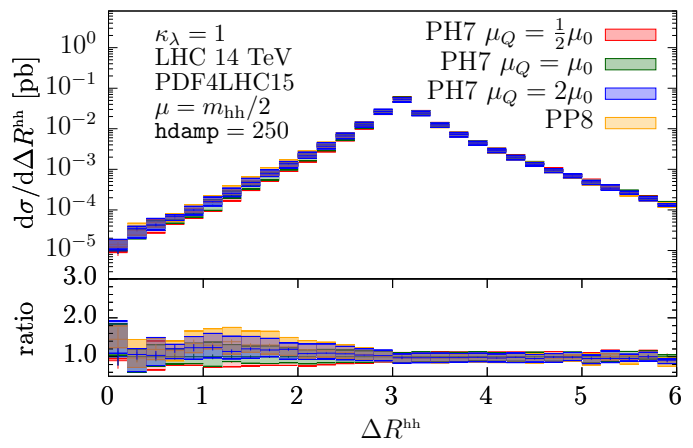

(b)

Figure 7. Higgs boson pair transverse momentum and $R$-separation for variations of the Herwig $\tilde{q}$-shower hard scale.

\section{Conclusions}

We have presented results for Higgs boson pair production in gluon fusion at full NLO QCD for non-standard values of the trilinear Higgs boson coupling $\lambda$. We have also shown how results with a modified top quark Yukawa coupling can be produced with the same code. We have demonstrated that the dependence of both the total and the differential $\mathrm{K}$-factors on the value of $\lambda$ is stronger than the $m_{t} \rightarrow \infty$ limit may suggest. The total cross section is a quadratic polynomial in $\lambda$, with a minimum around $\kappa_{\lambda} \approx 2.4$, which is present both at LO and NLO with full top quark mass dependence, stemming from destructive interference of diagrams with and without a trilinear Higgs coupling. The $m_{\mathrm{hh}}$ distribution shows a dip around this minimum, which is to lesser extent also visible in the transverse momentum distribution of one of the Higgs bosons. We have assumed in our study that modifications of the Higgs couplings to other particles are small and can be increasingly well constrained by other processes. Nonetheless, it should be kept in mind that a dip in the $m_{\mathrm{hh}}$ distribution could also originate from other effective couplings, for example an effective $t \bar{t} H H$ coupling, while $\kappa_{\lambda}=1$ [14].

We have also combined our NLO QCD results with the Pythia 8.2 and Herwig7.1 parton showers. In the Herwig 7.1 case we employed both the default shower (the angularordered $\tilde{q}$-shower) and the dipole shower. We observed that for distributions particularly sensitive to the additional radiation, the parton showers exhibit a somewhat different behaviour. While both Herwig 7.1 showers generate comparable results and perform as expected in the NLO regime, the Pythia 8.2 shower produces harder radiation, for example in the tail of the $p_{T}^{\text {hh }}$ distribution. Varying the shower hard scale in Herwig 7.1 on top of $\mu_{R}, \mu_{F}$ variations leads to uncertainty bands which approximately cover these differences. However, the parton shower uncertainties can then become sizeable and even surpass the fixed-order scale uncertainties.

The POWHEG version of the code for Higgs boson pair production including the possibility to vary the trilinear coupling and the top quark Yukawa coupling is publicly available in the POWHEG-BOX-V2 package at the website http://powhegbox.mib.infn.it, in the User-Processes-V2/ggHH/ directory. 


\section{Acknowledgments}

We would like to thank Gerhard Buchalla, Alejandro Celis, Matteo Capozi, Stephan Jahn and Emmanuele Re for helpful discussions. This research was supported in part by the COST Action CA16201 'Particleface' of the European Union, and by the Swiss National Science Foundation (SNF) under grant number 200020-175595. We gratefully acknowledge resources provided by the Max Planck Computing and Data Facility (MPCDF).

Open Access. This article is distributed under the terms of the Creative Commons Attribution License (CC-BY 4.0), which permits any use, distribution and reproduction in any medium, provided the original author(s) and source are credited.

\section{References}

[1] ATLAS, CMS collaboration, Measurements of the Higgs boson production and decay rates and constraints on its couplings from a combined ATLAS and CMS analysis of the LHC pp collision data at $\sqrt{s}=7$ and $8 \mathrm{TeV}, J H E P 08$ (2016) 045 [arXiv: 1606. 02266] [INSPIRE].

[2] ATLAS collaboration, Measurement of the Higgs boson coupling properties in the $H \rightarrow Z Z^{*} \rightarrow 4 \ell$ decay channel at $\sqrt{s}=13 \mathrm{TeV}$ with the ATLAS detector, JHEP 03 (2018) 095 [arXiv: 1712.02304] [INSPIRE].

[3] ATLAS collaboration, Combined measurements of Higgs boson production and decay using up to $80 \mathrm{fb}^{-1}$ of proton-proton collision data at $\sqrt{\mathrm{s}}=13 \mathrm{TeV}$ collected with the ATLAS experiment, ATLAS-CONF-2019-005 (2019).

[4] CMS collaboration, Combined measurements of Higgs boson couplings in proton-proton collisions at $\sqrt{s}=13 \mathrm{TeV}$, Eur. Phys. J. C 79 (2019) 421 [arXiv:1809.10733] [INSPIRE].

[5] CMS collaboration, Measurement and interpretation of differential cross sections for Higgs boson production at $\sqrt{s}=13 \mathrm{TeV}$, Phys. Lett. B 792 (2019) 369 [arXiv:1812.06504] [INSPIRE].

[6] J. Baglio et al., The measurement of the Higgs self-coupling at the LHC: theoretical status, JHEP 04 (2013) 151 [arXiv:1212.5581] [INSPIRE].

[7] R. Frederix et al., Higgs pair production at the LHC with NLO and parton-shower effects, Phys. Lett. B 732 (2014) 142 [arXiv:1401.7340] [INSPIRE].

[8] CMS collaboration, Combination of searches for Higgs boson pair production in proton-proton collisions at $\sqrt{s}=13$ TeV, Phys. Rev. Lett. 122 (2019) 121803 [arXiv: 1811.09689] [INSPIRE].

[9] CMS collaboration, Search for Higgs boson pair production in the $\gamma \gamma b \bar{b}$ final state in $p p$ collisions at $\sqrt{s}=13 \mathrm{TeV}$, Phys. Lett. B 788 (2019) 7 [arXiv:1806.00408] [INSPIRE].

[10] ATLAS collaboration, Combination of searches for Higgs boson pairs in pp collisions at 13 TeV with the ATLAS experiment., ATLAS-CONF-2018-043 (2018).

[11] ATLAS collaboration, Search for Higgs boson pair production in the $\gamma \gamma b \bar{b}$ final state with 13 TeV pp collision data collected by the ATLAS experiment, JHEP 11 (2018) 040 [arXiv: 1807.04873] [INSPIRE].

[12] A. Carvalho et al., Higgs pair production: choosing benchmarks with cluster analysis, JHEP 04 (2016) 126 [arXiv:1507.02245] [INSPIRE]. 
[13] A. Carvalho et al., Analytical parametrization and shape classification of anomalous $H H$ production in the EFT approach, arXiv:1608.06578 [INSPIRE].

[14] G. Buchalla et al., Higgs boson pair production in non-linear effective field theory with full $m_{t}$-dependence at NLO QCD, JHEP 09 (2018) 057 [arXiv:1806.05162] [INSPIRE].

[15] R. Gröber, M. Mühlleitner, M. Spira and J. Streicher, NLO QCD corrections to Higgs pair production including dimension-6 operators, JHEP 09 (2015) 092 [arXiv:1504.06577] [INSPIRE].

[16] R. Gröber, M. Mühlleitner and M. Spira, Higgs pair production at NLO QCD for CP-violating Higgs sectors, Nucl. Phys. B 925 (2017) 1 [arXiv:1705.05314] [InSPIRE].

[17] D. de Florian, I. Fabre and J. Mazzitelli, Higgs boson pair production at NNLO in QCD including dimension 6 operators, JHEP 10 (2017) 215 [arXiv:1704.05700] [INSPIRE].

[18] A. Falkowski and R. Rattazzi, Which EFT, arXiv:1902.05936 [InSPIRE].

[19] S. Chang and M.A. Luty, The Higgs trilinear coupling and the scale of new physics, arXiv: 1902.05556 [INSPIRE].

[20] L. Di Luzio, R. Gröber and M. Spannowsky, Maxi-sizing the trilinear Higgs self-coupling: how large could it be?, Eur. Phys. J. C 77 (2017) 788 [arXiv:1704.02311] [INSPIRE].

[21] S. Di Vita et al., A global view on the Higgs self-coupling, JHEP 09 (2017) 069 [arXiv: 1704.01953] [INSPIRE].

[22] J. Braathen and S. Kanemura, On two-loop corrections to the Higgs trilinear coupling in models with extended scalar sectors, arXiv:1903.05417 [INSPIRE].

[23] P. Basler, S. Dawson, C. Englert and M. Mühlleitner, Showcasing HH production: benchmarks for the LHC and HL-LHC, Phys. Rev. D 99 (2019) 055048 [arXiv: 1812.03542] [INSPIRE].

[24] K.S. Babu and S. Jana, Enhanced di-Higgs production in the two Higgs doublet model, JHEP 02 (2019) 193 [arXiv:1812.11943] [INSPIRE].

[25] I.M. Lewis and M. Sullivan, Benchmarks for double Higgs production in the singlet extended standard model at the LHC, Phys. Rev. D 96 (2017) 035037 [arXiv:1701.08774] [InSPIRE].

[26] Physics of the HL-LHC Working Group collaboration, Higgs physics at the HL-LHC and $H E-L H C$, arXiv: 1902.00134 [INSPIRE].

[27] S. Homiller and P. Meade, Measurement of the triple Higgs coupling at a HE-LHC, JHEP 03 (2019) 055 [arXiv: 1811.02572] [INSPIRE].

[28] W. Bizoń, U. Haisch and L. Rottoli, Constraints on the quartic Higgs self-coupling from double-Higgs production at future hadron colliders, arXiv:1810.04665 [INSPIRE].

[29] D. Gonçalves et al., Higgs boson pair production at future hadron colliders: from kinematics to dynamics, Phys. Rev. D 97 (2018) 113004 [arXiv: 1802.04319] [INSPIRE].

[30] J.H. Kim, Y. Sakaki and M. Son, Combined analysis of double Higgs production via gluon fusion at the HL-LHC in the effective field theory approach, Phys. Rev. D 98 (2018) 015016 [arXiv: 1801.06093] [INSPIRE].

[31] A. Adhikary et al., Revisiting the non-resonant Higgs pair production at the HL-LHC, JHEP 07 (2018) 116 [arXiv:1712.05346] [INSPIRE]. 
[32] A. Alves, T. Ghosh and K. Sinha, Can we discover double Higgs production at the LHC?, Phys. Rev. D 96 (2017) 035022 [arXiv: 1704.07395] [InSPIRE].

[33] Q.-H. Cao, G. Li, B. Yan, D.-M. Zhang and H. Zhang, Double Higgs production at the $14 \mathrm{TeV}$ LHC and a $100 \mathrm{TeV}$ pp collider, Phys. Rev. D 96 (2017) 095031 [arXiv: 1611.09336] [INSPIRE].

[34] A. Azatov, R. Contino, G. Panico and M. Son, Effective field theory analysis of double Higgs boson production via gluon fusion, Phys. Rev. D 92 (2015) 035001 [arXiv: 1502.00539] [INSPIRE].

[35] G. Li, L. X. Xu, B. Yan and C.P. Yuan, Resolving the degeneracy in top quark Yukawa coupling with Higgs pair production, arXiv:1904.12006 [INSPIRE].

[36] T. Corbett, A. Joglekar, H.L. Li and J.H. Yu, Exploring extended scalar sectors with di-Higgs signals: a Higgs EFT perspective, JHEP 05 (2018) 061 [arXiv:1705.02551] [INSPIRE].

[37] O.J.P. Eboli, G.C. Marques, S.F. Novaes and A.A. Natale, TWIN higgs boson production, Phys. Lett. B 197 (1987) 269 [INSPIRE].

[38] E.W.N. Glover and J.J. van der Bij, Higgs boson pair production via gluon fusion, Nucl. Phys. B 309 (1988) 282 [inSPIRE].

[39] T. Plehn, M. Spira and P.M. Zerwas, Pair production of neutral Higgs particles in gluon-gluon collisions, Nucl. Phys. B 479 (1996) 46 [Erratum ibid. B 531 (1998) 655] [hep-ph/9603205] [INSPIRE].

[40] S. Borowka et al., Higgs boson pair production in gluon fusion at next-to-leading order with full top-quark mass dependence, Phys. Rev. Lett. 117 (2016) 012001 [Erratum ibid. 117 (2016) 079901] [arXiv: 1604.06447] [INSPIRE].

[41] S. Borowka et al., Full top quark mass dependence in Higgs boson pair production at NLO, JHEP 10 (2016) 107 [arXiv:1608.04798] [INSPIRE].

[42] J. Baglio et al., Gluon fusion into Higgs pairs at NLO QCD and the top mass scheme, arXiv: 1811.05692 [INSPIRE].

[43] G. Ferrera and J. Pires, Transverse-momentum resummation for Higgs boson pair production at the LHC with top-quark mass effects, JHEP 02 (2017) 139 [arXiv: 1609.01691] [INSPIRE].

[44] G. Heinrich et al., NLO predictions for Higgs boson pair production with full top quark mass dependence matched to parton showers, JHEP 08 (2017) 088 [arXiv:1703.09252] [INSPIRE].

[45] S. Jones and S. Kuttimalai, Parton shower and NLO-matching uncertainties in Higgs boson pair production, JHEP 02 (2018) 176 [arXiv:1711.03319] [INSPIRE].

[46] S. Dawson, S. Dittmaier and M. Spira, Neutral Higgs boson pair production at hadron colliders: QCD corrections, Phys. Rev. D 58 (1998) 115012 [hep-ph/9805244] [INSPIRE].

[47] F. Maltoni, E. Vryonidou and M. Zaro, Top-quark mass effects in double and triple Higgs production in gluon-gluon fusion at NLO, JHEP 11 (2014) 079 [arXiv:1408.6542] [INSPIRE].

[48] D. de Florian and J. Mazzitelli, Two-loop virtual corrections to Higgs pair production, Phys. Lett. B 724 (2013) 306 [arXiv:1305.5206] [INSPIRE]. 
[49] D. de Florian and J. Mazzitelli, Higgs boson pair production at next-to-next-to-leading order in QCD, Phys. Rev. Lett. 111 (2013) 201801 [arXiv:1309.6594] [INSPIRE].

[50] J. Grigo, K. Melnikov and M. Steinhauser, Virtual corrections to Higgs boson pair production in the large top quark mass limit, Nucl. Phys. B 888 (2014) 17 [arXiv: 1408.2422] [INSPIRE].

[51] D. de Florian et al., Differential Higgs boson pair production at next-to-next-to-leading order in QCD, JHEP 09 (2016) 151 [arXiv:1606.09519] [INSPIRE].

[52] J. Grigo, J. Hoff and M. Steinhauser, Higgs boson pair production: top quark mass effects at NLO and NNLO, Nucl. Phys. B 900 (2015) 412 [arXiv: 1508.00909] [inSPIRE].

[53] D. de Florian and J. Mazzitelli, Higgs pair production at next-to-next-to-leading logarithmic accuracy at the LHC, JHEP 09 (2015) 053 [arXiv: 1505.07122] [INSPIRE].

[54] M. Grazzini et al., Higgs boson pair production at NNLO with top quark mass effects, JHEP 05 (2018) 059 [arXiv: 1803. 02463] [INSPIRE].

[55] D. De Florian and J. Mazzitelli, Soft gluon resummation for Higgs boson pair production including finite $M_{t}$ effects, JHEP 08 (2018) 156 [arXiv: 1807.03704] [INSPIRE].

[56] R. Gröber, A. Maier and T. Rauh, Reconstruction of top-quark mass effects in Higgs pair production and other gluon-fusion processes, JHEP 03 (2018) 020 [arXiv: 1709.07799] [INSPIRE].

[57] R. Bonciani, G. Degrassi, P.P. Giardino and R. Gröber, Analytical method for next-to-leading-order QCD corrections to double-Higgs production, Phys. Rev. Lett. 121 (2018) 162003 [arXiv: 1806.11564] [INSPIRE].

[58] X. Xu and L.L. Yang, Towards a new approximation for pair-production and associated-production of the Higgs boson, JHEP 01 (2019) 211 [arXiv:1810.12002] [INSPIRE].

[59] J. Davies, G. Mishima, M. Steinhauser and D. Wellmann, Double-Higgs boson production in the high-energy limit: planar master integrals, JHEP 03 (2018) 048 [arXiv:1801.09696] [INSPIRE].

[60] J. Davies, G. Mishima, M. Steinhauser and D. Wellmann, Double Higgs boson production at NLO in the high-energy limit: complete analytic results, JHEP 01 (2019) 176 [arXiv: 1811.05489] [INSPIRE].

[61] J. Davies, F. Herren, G. Mishima and M. Steinhauser, Real-virtual corrections to Higgs boson pair production at NNLO: three closed top quark loops, JHEP 05 (2019) 157 [arXiv: 1904.11998] [INSPIRE].

[62] S. Dawson, C. Englert and T. Plehn, Higgs physics: it ain't over till it's over, arXiv: 1808.01324 [INSPIRE].

[63] G. Brooijmans et al., Les Houches 2017: physics at TeV colliders new physics working group report, arXiv: 1803.10379 [INSPIRE].

[64] LHC Higgs Cross Section Working Group collaboration, Handbook of LHC Higgs Cross Sections: 4. Deciphering the nature of the Higgs sector, arXiv:1610.07922 [INSPIRE].

[65] M. Gorbahn and U. Haisch, Two-loop amplitudes for Higgs plus jet production involving a modified trilinear Higgs coupling, JHEP 04 (2019) 062 [arXiv: 1902.05480] [INSPIRE]. 
[66] J. Nakamura and A. Shivaji, Direct measurement of the Higgs self-coupling in $e^{+} e^{-} \rightarrow Z H$, arXiv: 1812.01576 [INSPIRE].

[67] S. Borowka et al., Probing the scalar potential via double Higgs boson production at hadron colliders, JHEP 04 (2019) 016 [arXiv:1811.12366] [INSPIRE].

[68] W. Kilian, S. Sun, Q.-S. Yan, X. Zhao and Z. Zhao, Multi-Higgs production and unitarity in vector-boson fusion at future hadron colliders, arXiv:1808.05534 [INSPIRE].

[69] E. Vryonidou and C. Zhang, Dimension-six electroweak top-loop effects in Higgs production and decay, JHEP 08 (2018) 036 [arXiv:1804.09766] [INSPIRE].

[70] F. Maltoni, D. Pagani and X. Zhao, Constraining the Higgs self-couplings at $e^{+} e^{-}$colliders, JHEP 07 (2018) 087 [arXiv: 1802.07616] [INSPIRE].

[71] F. Maltoni, D. Pagani, A. Shivaji and X. Zhao, Trilinear Higgs coupling determination via single-Higgs differential measurements at the LHC, Eur. Phys. J. C 77 (2017) 887 [arXiv: 1709.08649] [INSPIRE].

[72] G.D. Kribs, A. Maier, H. Rzehak, M. Spannowsky and P. Waite, Electroweak oblique parameters as a probe of the trilinear Higgs boson self-interaction, Phys. Rev. D 95 (2017) 093004 [arXiv: 1702.07678] [INSPIRE].

[73] G. Degrassi, M. Fedele and P.P. Giardino, Constraints on the trilinear Higgs self coupling from precision observables, JHEP 04 (2017) 155 [arXiv: 1702.01737] [INSPIRE].

[74] W. Bizon, M. Gorbahn, U. Haisch and G. Zanderighi, Constraints on the trilinear Higgs coupling from vector boson fusion and associated Higgs production at the LHC, JHEP 07 (2017) 083 [arXiv: 1610.05771] [INSPIRE].

[75] G. Degrassi, P.P. Giardino, F. Maltoni and D. Pagani, Probing the Higgs self coupling via single Higgs production at the LHC, JHEP 12 (2016) 080 [arXiv:1607.04251] [INSPIRE].

[76] M. Gorbahn and U. Haisch, Indirect probes of the trilinear Higgs coupling: $g g \rightarrow h$ and $h \rightarrow \gamma \gamma$, JHEP 10 (2016) 094 [arXiv: 1607.03773] [INSPIRE].

[77] M. McCullough, An indirect model-dependent probe of the Higgs self-coupling, Phys. Rev. D 90 (2014) 015001 [Erratum ibid. D 92 (2015) 039903] [arXiv: 1312.3322] [INSPIRE].

[78] P. Nason, A new method for combining NLO QCD with shower Monte Carlo algorithms, JHEP 11 (2004) 040 [hep-ph/0409146] [INSPIRE].

[79] S. Frixione, P. Nason and C. Oleari, Matching NLO QCD computations with Parton Shower simulations: the POWHEG method, JHEP 11 (2007) 070 [arXiv:0709.2092] [INSPIRE].

[80] S. Alioli, P. Nason, C. Oleari and E. Re, A general framework for implementing NLO calculations in shower Monte Carlo programs: the POWHEG BOX, JHEP 06 (2010) 043 [arXiv: 1002 .2581] [INSPIRE].

[81] T. Sjöstrand et al., An introduction to PYTHIA 8.2, Comput. Phys. Commun. 191 (2015) 159 [arXiv: 1410.3012] [inSPIRE].

[82] J. Bellm et al., HERWIG 7.1 release note, arXiv:1705.06919 [INSPIRE].

[83] G. Luisoni, P. Nason, C. Oleari and F. Tramontano, $H W^{ \pm} / H Z+0$ and 1 jet at NLO with the POWHEG BOX interfaced to GoSam and their merging within MiNLO, JHEP 10 (2013) 083 [arXiv: 1306.2542] [INSPIRE].

[84] G. Cullen et al., Automated one-loop calculations with GoSam, Eur. Phys. J. C 72 (2012) 1889 [arXiv:1111.2034] [INSPIRE]. 
[85] G. Cullen et al., GoSam-2.0: a tool for automated one-loop calculations within the Standard Model and beyond, Eur. Phys. J. C 74 (2014) 3001 [arXiv:1404.7096] [InSPIRE].

[86] T. Peraro, Ninja: automated integrand reduction via Laurent expansion for one-loop amplitudes, Comput. Phys. Commun. 185 (2014) 2771 [arXiv:1403.1229] [INSPIRE].

[87] T. Binoth et al., Golem95: a numerical program to calculate one-loop tensor integrals with up to six external legs, Comput. Phys. Commun. 180 (2009) 2317 [arXiv:0810.0992] [INSPIRE].

[88] G. Cullen et al., Golem95C: a library for one-loop integrals with complex masses, Comput. Phys. Commun. 182 (2011) 2276 [arXiv:1101.5595] [InSPIRE].

[89] A. van Hameren, OneLOop: for the evaluation of one-loop scalar functions, Comput. Phys. Commun. 182 (2011) 2427 [arXiv:1007.4716] [INSPIRE].

[90] K. Arnold et al., VBFNLO: a parton level Monte Carlo for processes with electroweak bosons, Comput. Phys. Commun. 180 (2009) 1661 [arXiv:0811.4559] [INSPIRE].

[91] J. Baglio et al., Release Note - VBFNLO 2.7.0, arXiv:1404.3940 [INSPIRE].

[92] A. von Manteuffel and C. Studerus, Reduze 2 - Distributed Feynman Integral Reduction, arXiv: 1201.4330 [INSPIRE].

[93] S. Borowka et al., SecDec-3.0: numerical evaluation of multi-scale integrals beyond one loop, Comput. Phys. Commun. 196 (2015) 470 [arXiv:1502.06595] [INSPIRE].

[94] J. Butterworth et al., PDF 4 LHC recommendations for LHC Run II, J. Phys. G 43 (2016) 023001 [arXiv: 1510.03865] [INSPIRE].

[95] S. Dulat et al., New parton distribution functions from a global analysis of quantum chromodynamics, Phys. Rev. D 93 (2016) 033006 [arXiv:1506.07443] [InSPIRE].

[96] L.A. Harland-Lang, A.D. Martin, P. Motylinski and R.S. Thorne, Parton distributions in the LHC era: MMHT 2014 PDFs, Eur. Phys. J. C 75 (2015) 204 [arXiv:1412.3989] [INSPIRE].

[97] NNPDF collaboration, Parton distributions for the LHC Run II, JHEP 04 (2015) 040 [arXiv:1410.8849] [INSPIRE].

[98] A. Buckley et al., LHAPDF6: parton density access in the LHC precision era, Eur. Phys. J. C 75 (2015) 132 [arXiv:1412.7420] [INSPIRE].

[99] M. Cacciari, G.P. Salam and G. Soyez, The anti-k $k_{t}$ jet clustering algorithm, JHEP 04 (2008) 063 [arXiv: 0802.1189] [InSPIRE].

[100] M. Cacciari and G.P. Salam, Dispelling the $N^{3}$ myth for the $k_{t}$ jet-finder, Phys. Lett. B 641 (2006) 57 [hep-ph/0512210] [INSPIRE].

[101] M. Cacciari, G.P. Salam and G. Soyez, FastJet user manual, Eur. Phys. J. C 72 (2012) 1896 [arXiv: 1111.6097] [inSPIRE].

[102] S. Ferrario Ravasio, T. Ježo, P. Nason and C. Oleari, A theoretical study of top-mass measurements at the LHC using NLO+PS generators of increasing accuracy, Eur. Phys. J. C 78 (2018) 458 [arXiv:1801.03944] [InSPIRE].

[103] J.R. Andersen et al., Les Houches 2017: physics at TeV colliders standard model working group report, arXiv: 1803.07977. 